\title{
Bio-efficacy of Seed Protectant Chemicals for Management of Angoumois Grain Moth on Paddy
}

\author{
Basavanjali $^{1 *}$, Sushila Nadagouda ${ }^{1}$, A. Prabhuraj ${ }^{1}$, Shivaleela $^{1}$ and Basavegowda ${ }^{2}$ \\ ${ }^{1}$ Department of Agricultural Entomology, College of Agriculture, Raichur, India \\ ${ }^{2}$ Seed Unit, University of Agricultural Sciences, Raichur, 584104, India \\ *Corresponding author
}

\section{A B S T R A C T}

Keywords

Spinosad,

Emamectin

benzoate, Seed

damage, Paddy and

angoumois grain

moth

Article Info

Accepted:

07 September 2019

Available Online:

10 October 2019
Studies on the bio-efficacy of seed protectant chemicals against angoumois grain moth on paddy was carried out in the Seed unit UAS, Raichur during 2018-19 with ambient conditions $\left(20.3 \pm 2.3{ }^{\circ} \mathrm{C}\right.$ temperature and $50.5 \pm 6.3$ $\% \mathrm{RH})$. The observations were recorded on seed damage, seed weight loss and live adults up to 180 DAT. Up to ninety days after treatment imposition no seed damage, no seed weight loss and no live adults was noticed in any of the treatments. The treatments of spinosad $45 \mathrm{SC} @ 4.4 \mathrm{mg} \mathrm{kg}^{-1}$ and emamectin benzoate $5 \mathrm{SG} @ 40 \mathrm{mg} \mathrm{kg}^{-1}$ recorded least seed damage, least seed weight loss and suppression of adult population at one hundred and eighty days of treatment imposition and both differed significantly from remaining treatments.

\section{Introduction}

Rice is a vital food to more than half of the world's population. Rice accounts for 55 per cent of total cereal production in the country. In India rice is grown over 42.9 million ha, with production of $111.1 \mathrm{MT}$ and productivity of $2580 \mathrm{~kg}$ per ha (Anon., 2018). Rice provides instant energy as its most important component is carbohydrate (starch) and fat content or lipids (1\%) and due to this reason it is considered as a complete food. After harvesting, unprocessed paddy is stored for varied periods of time depending on market demand, size of production and the farmer's needs. Storage is the most important and critical postharvest operation.

Generally lepidopterans and coleopterans cause maximum damage in storage (Usman, 1957). In India the annual storage losses were estimated as 14 million tonnes of food grains worth $\$ 16,000$ million every year.

Out of this, food grain losses due to insects alone account for a monetary loss of $\$ 300$ 
million (Mohan and Kavitharaghavan, 2008). During storage, paddy is highly vulnerable to infestation by a variety of insect pests and diseases.

Among them, the Angoumois grain moth, Sitotroga cerealella (Olivier) was the most destructive internal pests of paddy and it is carried over from field to the storehouses through the field infested grains.

Therefore, there is an urgent need to identify insecticides that can effectively prevent the storage losses, easily available, affordable, safer and least detrimental to the environment.

Many of these insecticides are effective at relatively low dosage and provide long term protection, which can range from six to twelve months (Athanassiou, 2004). In the countries where storage facilities are inadequate, insecticides are one of the most effective weapons for disinfesting and protecting stored products from infestation.

\section{Materials and Methods}

Studies on the bio-efficacy of seed protectant chemicals like emamectin benzoate 5 SG @ $40 \mathrm{mg} \mathrm{kg}{ }^{-1}$, spinosad $45 \mathrm{SC}$ @ $4.4 \mathrm{mg} \mathrm{kg}^{-1}$, deltamethrin 2.8 EC @ $0.04 \mathrm{ml} \mathrm{kg}{ }^{-1}$, chlorfenapyr 10 EC @ $0.02 \mathrm{ml} \mathrm{kg}^{-1}$, acorus TNAU formulation@10 $\mathrm{g} \mathrm{kg}^{-1}$ and sweet flag rhizome powder @ $20 \mathrm{~g} \mathrm{~kg}^{-1}$ against angoumois grain moth on paddy was carried out in the Seed unit UAS, Raichur during 2018-19 with ambient conditions $(20.3 \pm 2.3$ ${ }^{\circ} \mathrm{C}$ temperature and $50.5 \pm 6.3 \% \mathrm{RH}$ ).

\section{Bioefficacy studies on angoumois grain moth}

The experiment was conducted in completely randomized design (CRD) with seven treatments and three replications. Two $\mathrm{kg}$ of freshly harvested paddy (Gangavati sona) were procured from Seed unit with high percentage of germination $(97.00 \%)$ and low moisture content $(10.49 \%)$ was taken for each treatment. Recommended quantities of insecticides were diluted in ten $\mathrm{ml}$ of water and 10 per cent sticky material (gum acacia) was added to treat two $\mathrm{kg}$ of seed for proper coating. Similarly, control was maintained without any treatment for comparison.

After treatment, seeds were dried in shade and packed in two kg capacity non woven bag and kept for storage under ambient condition for a period of six months. Later ten pairs of adults were released into each non woven bag.

Observations were made on per cent seed damage, seed weight loss and the number of live adults in representative sample.

\section{Seed damage $(\%)$}

Seeds were thoroughly mixed and one hundred seeds were randomly drawn from each treatment and replications, seeds with exit holes were considered as damaged seeds and expressed as per cent seed damage.

\section{Seed damage (\%) \\ $=\frac{\text { Number of seeds damaged }}{\text { Total number of seeds in the sample }} \times 100$}

\section{Seed weight loss}

It was computed by following formula as suggested by Harris and Limbald (1978).

Seed weight loss $(\%)=\frac{\text { O.W }- \text { C.W }}{\text { O.W }} \times 100$

Where;

$\mathrm{O} . \mathrm{W}=$ Original weight on dry weight basis

C.W $=$ Current weight on dry weight basis 
Number of live adult insects per $100 \mathrm{~g}$ seeds

Number of live adult insects emerged from $100 \mathrm{~g}$ sample seed of each replication of the treatment was counted.

\section{Results and Discussion}

\section{Seed damage}

No seed damage was recorded in all the treatments at ninety days after treatment. After one hundred and eighty days of treatment the spinosad 45 SC $(0.33 \%)$ and emamectin benzoate $5 \mathrm{SG}(0.67 \%)$ recorded lowest seed damage and were found to be the most superior in reducing the insect damage among all other treatments and were on par with each other.

Highest (18.67\%) per cent seed damage was recorded in untreated control and was inferior to all the other treatments. The order of efficacy of different seed protectant chemicals was as follows spinosad $45 \mathrm{SC}=$ emamectin benzoate $5 \mathrm{SG}>$ acorus TNAU formulation $>$ deltamethrin 2.8 EC > sweet flag rhizome powder > chlorfenapyr $10 \mathrm{EC}$ (Table 1).

Table.1 Effect of seed protectant chemicals on seed damage of paddy seed by S. cerealella

\begin{tabular}{|c|c|c|c|c|c|c|c|c|}
\hline \multirow[t]{2}{*}{ Treatments } & \multirow{2}{*}{$\begin{array}{l}\text { Dosage/ } \\
\text { kg seed }\end{array}$} & \multicolumn{7}{|c|}{ Seed damage $(\%)$} \\
\hline & & 30 DAT & 60 DAT & 90 DAT & $\begin{array}{c}120 \\
\text { DAT }\end{array}$ & $\begin{array}{c}150 \\
\text { DAT }\end{array}$ & $\begin{array}{l}\text { 180 } \\
\text { DAT }\end{array}$ & Mean \\
\hline $\begin{array}{l}\mathrm{T}_{1} \text { - Emamectin } \\
\text { benzoate } 5 \mathrm{SG}\end{array}$ & $40 \mathrm{mg}$ & $\begin{array}{c}0.00 \\
(0.57)^{\mathrm{a}}\end{array}$ & $\begin{array}{c}0.00 \\
(0.57)^{\mathrm{a}}\end{array}$ & $\begin{array}{c}0.00 \\
(0.57)^{\mathrm{a}}\end{array}$ & $\begin{array}{c}0.00 \\
(0.57)^{\mathrm{a}}\end{array}$ & $\begin{array}{c}0.33 \\
(1.91)^{\mathrm{a}}\end{array}$ & $\begin{array}{c}0.67 \\
(3.83)^{\mathrm{a}}\end{array}$ & 0.17 \\
\hline $\begin{array}{c}T_{2}-\text { Spinosad } 45 \\
\text { SC }\end{array}$ & $4.4 \mathrm{mg}$ & $\begin{array}{c}0.00 \\
(0.57)^{\mathrm{a}}\end{array}$ & $\begin{array}{c}0.00 \\
(0.57)^{\mathrm{a}}\end{array}$ & $\begin{array}{c}0.00 \\
(0.57)^{\mathrm{a}}\end{array}$ & $\begin{array}{c}0.00 \\
(0.57)^{\mathrm{a}}\end{array}$ & $\begin{array}{c}0.00 \\
(0.57)^{\mathrm{a}}\end{array}$ & $\begin{array}{c}0.33 \\
(6.54)^{\mathrm{a}}\end{array}$ & 0.05 \\
\hline $\begin{array}{c}\mathrm{T}_{3}- \\
\text { Deltamethrin } \\
\text { 2.8EC }\end{array}$ & $0.04 \mathrm{ml}$ & $\begin{array}{c}0.00 \\
(0.57)^{\mathrm{a}}\end{array}$ & $\begin{array}{c}0.00 \\
(0.57)^{\mathrm{a}}\end{array}$ & $\begin{array}{c}0.00 \\
(0.57)^{\mathrm{a}}\end{array}$ & $\begin{array}{c}0.67 \\
(3.83)^{\mathrm{a}}\end{array}$ & $\begin{array}{c}2.00 \\
(8.13)^{\mathrm{b}}\end{array}$ & $\begin{array}{c}2.33 \\
(8.74)^{\mathrm{b}}\end{array}$ & 0.83 \\
\hline $\begin{array}{c}\text { T }_{4-} \\
\text { Chlorfenapyr } 10\end{array}$ & $0.02 \mathrm{ml}$ & $\begin{array}{c}0.00 \\
(0.57)^{\mathrm{a}}\end{array}$ & $\begin{array}{c}0.00 \\
(0.57)^{\mathrm{a}}\end{array}$ & $\begin{array}{c}0.00 \\
(0.57)^{\mathrm{a}}\end{array}$ & $\begin{array}{c}1.00 \\
(5.74)^{\mathrm{a}}\end{array}$ & $\begin{array}{c}2.33 \\
(8.74)^{\mathrm{b}}\end{array}$ & $\begin{array}{c}3.00 \\
(9.88)^{b}\end{array}$ & 1.05 \\
\hline $\begin{array}{l}\mathrm{T}_{5} \text {-Acorus } \\
\text { TNAU } \\
\text { formulation }\end{array}$ & $10 \mathrm{~g}$ & $\begin{array}{r}0.00 \\
(0.57)^{\mathrm{a}}\end{array}$ & $\begin{array}{c}0.00 \\
(0.57)^{\mathrm{a}}\end{array}$ & $\begin{array}{c}0.00 \\
(0.57)^{\mathrm{a}}\end{array}$ & $\begin{array}{c}0.67 \\
(3.83)^{\mathrm{a}}\end{array}$ & $\begin{array}{c}1.67 \\
(7.33)^{\mathrm{b}}\end{array}$ & $\begin{array}{c}2.00 \\
(8.13)^{\mathrm{b}}\end{array}$ & 0.72 \\
\hline $\begin{array}{l}T_{6} \text {-Sweet flag } \\
\text { rhizome powder }\end{array}$ & $20 \mathrm{~g}$ & $\begin{array}{c}0.00 \\
(0.57)^{\mathrm{a}}\end{array}$ & $\begin{array}{c}0.00 \\
(0.57)^{\mathrm{a}}\end{array}$ & $\begin{array}{c}0.00 \\
(0.57)^{\mathrm{a}}\end{array}$ & $\begin{array}{c}0.67 \\
(3.83)^{\mathrm{a}}\end{array}$ & $\begin{array}{c}2.00 \\
(8.13)^{\mathrm{b}}\end{array}$ & $\begin{array}{c}2.33 \\
(8.74)^{\mathrm{b}}\end{array}$ & 0.83 \\
\hline $\begin{array}{c}\mathbf{T}_{7}-\text { Untreated } \\
\text { control }\end{array}$ & - & $\begin{array}{c}3.67 \\
(11.02)^{\mathrm{b}}\end{array}$ & $\begin{array}{c}5.33 \\
(13.34)^{\mathrm{b}}\end{array}$ & $\begin{array}{c}7.33 \\
(15.68)^{\mathrm{b}}\end{array}$ & $\begin{array}{c}11.00 \\
(19.36)^{\mathrm{b}}\end{array}$ & $\begin{array}{c}14.33 \\
(22.21)^{\mathrm{c}}\end{array}$ & $\begin{array}{r}18.67 \\
(25.60)^{\mathrm{c}}\end{array}$ & 10.05 \\
\hline S. Em \pm & & 0.20 & 0.16 & 0.28 & 1.14 & 0.84 & 1.05 & \\
\hline CD@ 1\% & & 0.83 & 0.67 & 1.19 & 4.82 & 3.55 & 4.42 & \\
\hline
\end{tabular}

DAT- Days after treatment

Figures in parentheses are arcsine transformed values

Figures in the column followed by same letters are not-significant at $\mathrm{p}=0.01$ by DMRT 
Table.2 Effect of seed protectant chemicals on seed weight loss of paddy by S. cerealella

\begin{tabular}{|c|c|c|c|c|c|c|c|c|c|}
\hline \multirow[t]{2}{*}{ Treatments } & \multirow{2}{*}{$\begin{array}{c}\text { Dosage } / \mathrm{Kg} \\
\text { seed }\end{array}$} & \multicolumn{8}{|c|}{ Seed weight loss (\%) } \\
\hline & & $\begin{array}{c}\text { 30 } \\
\text { DAT }\end{array}$ & $\begin{array}{c}\text { 60 } \\
\text { DAT }\end{array}$ & $\begin{array}{c}90 \\
\text { DAT }\end{array}$ & $\begin{array}{c}120 \\
\text { DAT }\end{array}$ & $\begin{array}{c}150 \\
\text { DAT }\end{array}$ & $\begin{array}{c}180 \\
\text { DAT }\end{array}$ & Mean & $\begin{array}{c}\text { Per cent } \\
\text { reduction } \\
\text { over } \\
\text { control }\end{array}$ \\
\hline $\begin{array}{c}\mathrm{T}_{1}- \\
\text { Emamectin } \\
\text { benzoate } 5 \mathrm{SG}\end{array}$ & $40 \mathrm{mg}$ & $\begin{array}{c}0.00 \\
(0.57)^{\mathrm{a}}\end{array}$ & $\begin{array}{c}0.00 \\
(0.57)^{\mathrm{a}}\end{array}$ & $\begin{array}{c}0.00 \\
(0.57)^{\mathrm{a}}\end{array}$ & $\begin{array}{c}0.08 \\
(1.57)^{\mathrm{a}}\end{array}$ & $\begin{array}{c}0.37 \\
(3.49)^{\mathrm{b}}\end{array}$ & $\begin{array}{c}0.47 \\
(3.86)^{\mathrm{b}}\end{array}$ & 0.15 & 98.32 \\
\hline $\begin{array}{l}\mathrm{T}_{2} \text {-Spinosad } \\
\text { 45 SC }\end{array}$ & $4.4 \mathrm{mg}$ & $\begin{array}{c}0.00 \\
(0.57)^{\mathrm{a}}\end{array}$ & $\begin{array}{c}0.00 \\
(0.57)^{\mathrm{a}}\end{array}$ & $\begin{array}{c}0.00 \\
(0.57)^{\mathrm{a}}\end{array}$ & $\begin{array}{c}0.00 \\
(0.57)^{\mathrm{a}}\end{array}$ & $\begin{array}{c}0.00 \\
(0.57)^{\mathrm{a}}\end{array}$ & $\begin{array}{c}0.23 \\
(2.77)^{\mathrm{a}}\end{array}$ & 0.03 & 99.66 \\
\hline $\begin{array}{c}\mathrm{T}_{3}- \\
\text { Deltamethrin } \\
2.8 \mathrm{EC}\end{array}$ & $0.04 \mathrm{ml}$ & $\begin{array}{c}0.00 \\
(0.57)^{\mathrm{a}}\end{array}$ & $\begin{array}{c}0.00 \\
(0.57)^{\mathrm{a}}\end{array}$ & $\begin{array}{c}0.00 \\
(0.57)^{\mathrm{a}}\end{array}$ & $\begin{array}{c}0.14 \\
(2.15)^{\mathrm{ab}}\end{array}$ & $\begin{array}{c}0.71 \\
(4.84)^{\mathrm{c}}\end{array}$ & $\begin{array}{c}1.40 \\
(6.79)^{\mathrm{c}}\end{array}$ & 0.38 & 95.70 \\
\hline $\begin{array}{c}\mathrm{T}_{4}- \\
\text { Chlorfenapyr } \\
\text { 10EC }\end{array}$ & $0.02 \mathrm{ml}$ & $\begin{array}{c}0.00 \\
(0.57)^{\mathrm{a}}\end{array}$ & $\begin{array}{c}0.00 \\
(0.57)^{\mathrm{a}}\end{array}$ & $\begin{array}{c}0.00 \\
(0.57)^{\mathrm{a}}\end{array}$ & $\begin{array}{c}0.32 \\
(3.22)^{\mathrm{b}}\end{array}$ & $\begin{array}{c}1.45 \\
(6.87)^{\mathrm{d}}\end{array}$ & $\begin{array}{c}2.02 \\
(8.18)^{\mathrm{e}}\end{array}$ & 0.63 & 92.95 \\
\hline $\begin{array}{c}T_{5} \text {-Acorus } \\
\text { TNAU } \\
\text { formulation }\end{array}$ & $10 \mathrm{~g}$ & $\begin{array}{c}0.00 \\
(0.57)^{\mathrm{a}}\end{array}$ & $\begin{array}{c}0.00 \\
(0.57)^{\mathrm{a}}\end{array}$ & $\begin{array}{c}0.00 \\
(0.57)^{\mathrm{a}}\end{array}$ & $\begin{array}{c}0.19 \\
(2.48)^{\mathrm{ab}}\end{array}$ & $\begin{array}{c}0.73 \\
(4.90)^{\mathrm{c}}\end{array}$ & $\begin{array}{c}1.53 \\
(7.10)^{\mathrm{bc}}\end{array}$ & 0.41 & 95.41 \\
\hline $\begin{array}{c}\mathbf{T}_{6}-\text {-Sweet flag } \\
\text { rhizome } \\
\text { powder }\end{array}$ & $20 \mathrm{~g}$ & $\begin{array}{c}0.00 \\
(0.57)^{\mathrm{a}}\end{array}$ & $\begin{array}{c}0.00 \\
(0.57)^{\mathrm{a}}\end{array}$ & $\begin{array}{c}0.00 \\
(0.57)^{\mathrm{a}}\end{array}$ & $\begin{array}{c}0.23 \\
(2.72)^{\mathrm{b}}\end{array}$ & $\begin{array}{c}0.79 \\
(5.08)^{\mathrm{c}}\end{array}$ & $\begin{array}{c}1.86 \\
(7.84)^{\mathrm{cd}}\end{array}$ & 0.48 & 94.63 \\
\hline $\begin{array}{c}T_{7}-\text { Untreated } \\
\text { control }\end{array}$ & - & $\begin{array}{c}2.34 \\
(8.81)^{\mathrm{b}}\end{array}$ & $\begin{array}{c}4.63 \\
(12.39)^{\mathrm{a}}\end{array}$ & $\begin{array}{c}7.83 \\
(16.24)^{b}\end{array}$ & $\begin{array}{c}9.43 \\
(17.88)^{\mathrm{c}}\end{array}$ & $\begin{array}{c}12.70 \\
(20.87)^{\mathrm{e}}\end{array}$ & $\begin{array}{l}16.68 \\
(24.10)^{\mathrm{f}}\end{array}$ & 8.94 & - \\
\hline S. Em \pm & & 0.02 & 0.02 & 0.14 & 0.24 & 0.23 & 0.22 & & \\
\hline CD@ 1\% & & 0.07 & 0.09 & 0.58 & 1.01 & 0.95 & 0.94 & & \\
\hline
\end{tabular}

DAT- Days after treatment

Figures in parentheses are arcsine transformed values

Figures in the column followed by same letters are not-significant at $\mathrm{p}=0.01$ by DMRT 
Table.3 Effect of seed protectant chemicals on emergence of adults of S. cerealella on paddy

\begin{tabular}{|c|c|c|c|c|c|c|c|c|}
\hline \multirow[t]{2}{*}{ Treatments } & \multirow{2}{*}{$\begin{array}{l}\text { Dosage/kg } \\
\text { seed }\end{array}$} & \multicolumn{7}{|c|}{ No of live adults / $100 \mathrm{~g}$ of seeds } \\
\hline & & $\begin{array}{c}\text { 30 } \\
\text { DAT }\end{array}$ & $\begin{array}{c}\text { 60 } \\
\text { DAT }\end{array}$ & $\begin{array}{l}90 \\
\text { DAT }\end{array}$ & $\begin{array}{l}120 \\
\text { DAT }\end{array}$ & $\begin{array}{l}\text { 150 } \\
\text { DAT }\end{array}$ & $\begin{array}{l}\text { 180 } \\
\text { DAT }\end{array}$ & Mean \\
\hline $\begin{array}{c}\mathrm{T}_{1} \text {-Emamectin benzoate } 5 \\
\text { SG }\end{array}$ & $40 \mathrm{mg}$ & $\begin{array}{c}0.00 \\
(0.71)^{\mathrm{a}}\end{array}$ & $\begin{array}{c}0.00 \\
(0.71)^{\mathrm{a}}\end{array}$ & $\begin{array}{c}0.00 \\
(0.71)^{\mathrm{a}}\end{array}$ & $\begin{array}{c}0.00 \\
(0.71)^{\mathrm{a}}\end{array}$ & $\begin{array}{c}1.00 \\
(1.17)^{\mathrm{ab}}\end{array}$ & $\begin{array}{c}2.00 \\
(1.56)^{\mathrm{ab}}\end{array}$ & 0.50 \\
\hline $\mathrm{T}_{2}$-Spinosad $45 \mathrm{SC}$ & $4.4 \mathrm{mg}$ & $\begin{array}{c}0.00 \\
(0.71)^{\mathrm{a}}\end{array}$ & $\begin{array}{c}0.00 \\
(0.71)^{\mathrm{a}}\end{array}$ & $\begin{array}{c}0.00 \\
(0.71)^{\mathrm{a}}\end{array}$ & $\begin{array}{c}0.00 \\
(0.71)^{\mathrm{a}}\end{array}$ & $\begin{array}{c}0.00 \\
(0.71)^{\mathrm{a}}\end{array}$ & $\begin{array}{c}1.00 \\
(1.17)^{\mathrm{a}}\end{array}$ & 0.16 \\
\hline $\mathrm{T}_{3}$-Deltamethrin 2.8EC & $0.04 \mathrm{ml}$ & $\begin{array}{c}0.00 \\
(0.71)^{\mathrm{a}}\end{array}$ & $\begin{array}{c}0.00 \\
(0.71)^{\mathrm{a}}\end{array}$ & $\begin{array}{c}0.00 \\
(0.71)^{\mathrm{a}}\end{array}$ & $\begin{array}{c}1.33 \\
(1.34)^{\mathrm{b}}\end{array}$ & $\begin{array}{c}1.67 \\
(1.46)^{b}\end{array}$ & $\begin{array}{l}3.33 \\
(2.12)^{\mathrm{b}}\end{array}$ & 1.05 \\
\hline $\mathrm{T}_{4}$-Chlorfenapyr $10 \mathrm{EC}$ & $0.02 \mathrm{ml}$ & $\begin{array}{c}0.00 \\
(0.71)^{\mathrm{a}}\end{array}$ & $\begin{array}{c}0.00 \\
(0.71)^{\mathrm{a}}\end{array}$ & $\begin{array}{c}0.00 \\
(0.71)^{\mathrm{a}}\end{array}$ & $\begin{array}{c}1.67 \\
(1.46)^{b}\end{array}$ & $\begin{array}{c}2.00 \\
(1.56)^{\mathrm{b}}\end{array}$ & $\begin{array}{c}4.67 \\
(2.35)^{\mathrm{bc}}\end{array}$ & 1.40 \\
\hline $\begin{array}{l}\mathrm{T}_{5} \text {-Acorus TNAU } \\
\text { formulation }\end{array}$ & $10 \mathrm{~g}$ & $\begin{array}{c}0.00 \\
(0.71)^{\mathrm{a}}\end{array}$ & $\begin{array}{c}0.00 \\
(0.71)^{\mathrm{a}}\end{array}$ & $\begin{array}{c}0.00 \\
(0.71)^{\mathrm{a}}\end{array}$ & $\begin{array}{c}1.33 \\
(1.34)^{\mathrm{b}}\end{array}$ & $\begin{array}{c}2.33 \\
(1.68)^{\mathrm{b}}\end{array}$ & $\begin{array}{c}4.00 \\
(1.87)^{\mathrm{b}}\end{array}$ & 1.27 \\
\hline $\begin{array}{l}\mathrm{T}_{6} \text {-Sweet flag rhizome } \\
\text { powder (A. calamus) }\end{array}$ & $20 \mathrm{~g}$ & $\begin{array}{c}0.00 \\
(0.71)^{\mathrm{a}}\end{array}$ & $\begin{array}{c}0.00 \\
(0.71)^{\mathrm{a}}\end{array}$ & $\begin{array}{c}0.00 \\
(0.71)^{\mathrm{a}}\end{array}$ & $\begin{array}{c}1.67 \\
(1.46)^{b}\end{array}$ & $\begin{array}{c}2.67 \\
(1.77)^{\mathrm{b}}\end{array}$ & $\begin{array}{c}4.33 \\
(2.20)^{\mathrm{bc}}\end{array}$ & 1.44 \\
\hline $\mathrm{T}_{7}$-Untreated control & ---- & $\begin{array}{c}7.67 \\
(2.86)^{b}\end{array}$ & $\begin{array}{l}12.67 \\
(3.63)^{b}\end{array}$ & $\begin{array}{c}18.33 \\
(4.34)^{b}\end{array}$ & $\begin{array}{l}25.67 \\
(5.12)^{\mathrm{c}}\end{array}$ & $\begin{array}{l}29.67 \\
(5.49)^{\mathrm{c}}\end{array}$ & $\begin{array}{l}32.33 \\
(5.73)^{\mathrm{c}}\end{array}$ & 21.05 \\
\hline S. Em \pm & & 0.02 & 0.02 & 0.01 & 0.09 & 0.14 & 0.14 & \\
\hline CD@1\% & & 0.09 & 0.07 & 0.06 & 0.38 & 0.58 & 0.59 & \\
\hline
\end{tabular}

DAT- Days after treatment; Figures in parentheses are $\sqrt{ }(\mathrm{x}+0.5)$ transformed value

Figures in the column followed by same letters are not-significant at $\mathrm{p}=0.01$ by DMRT

Fig.1 Effect of seed protectant chemicals on seed weight loss of paddy by S. cerealella

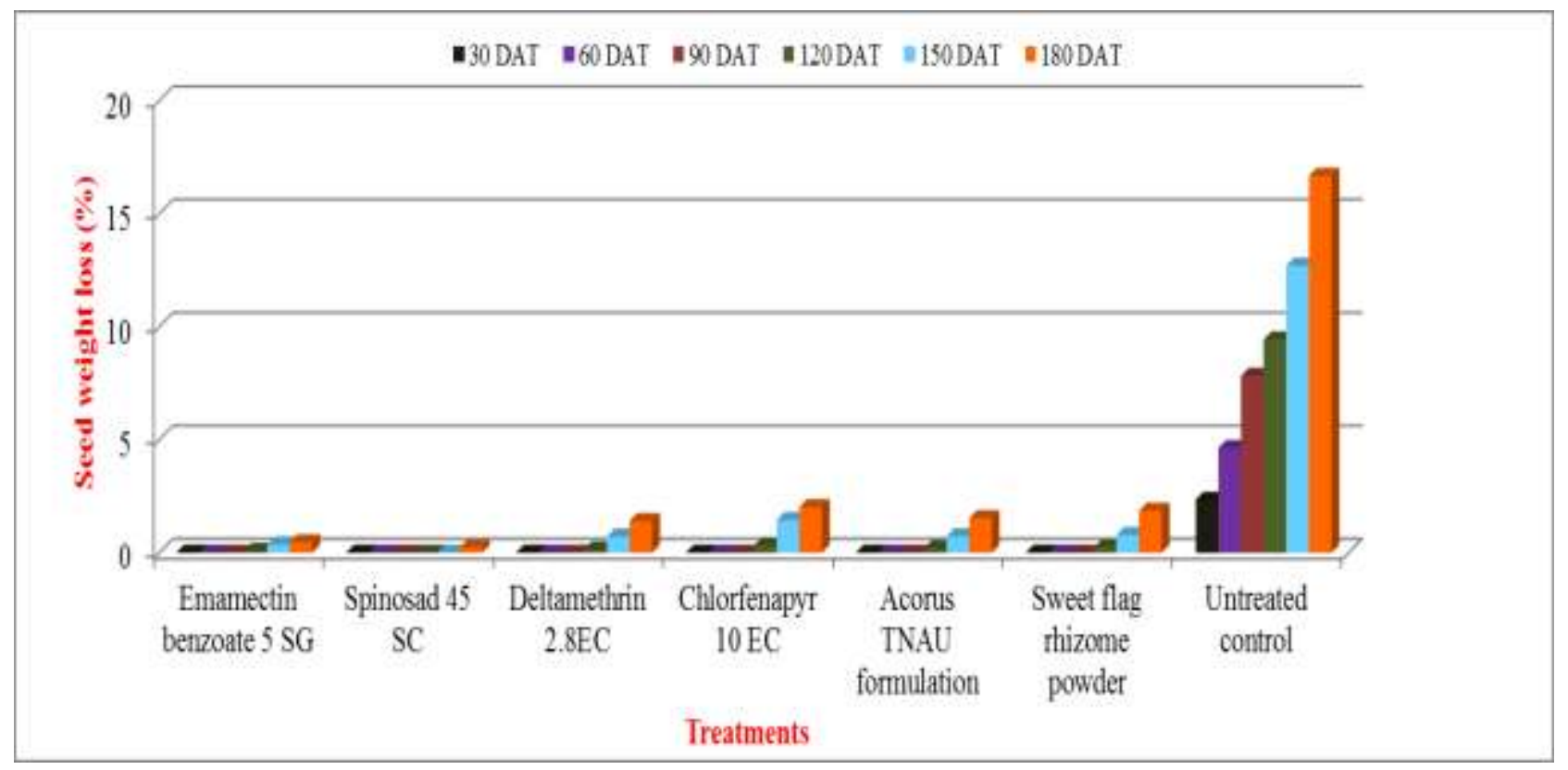


Fig.2 Effect of seed protectant chemicals on emergence of adults of S. cerealella on paddy

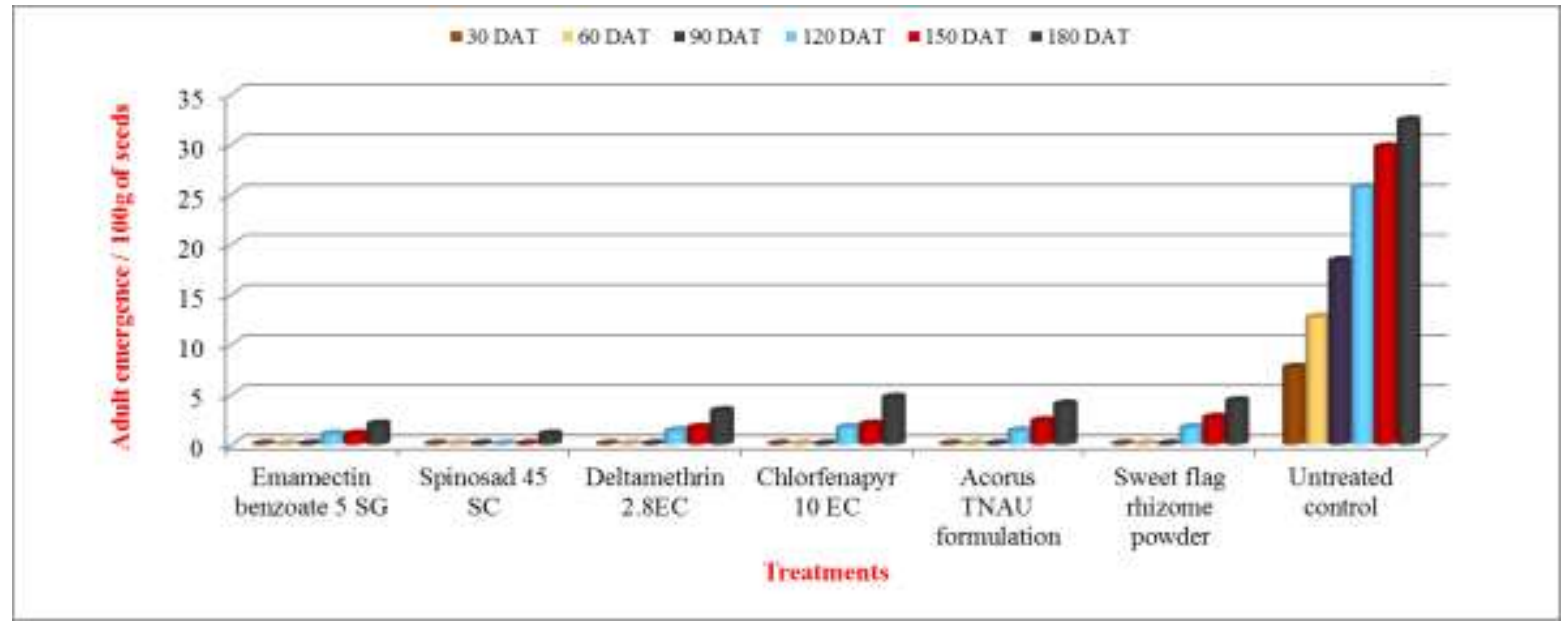

The spinosad having specific mode of action of spinosad is to alter the function of nicotinic and GABA-gated ion channels, causing rapid excitation of the insect nervous system, leading to involuntary muscle contractions, tremors, paralysis and death of the insect takes place and the emamectin benzoate does stimulation of high-affinity GABA receptors and a consequent increase in membrane chloride ion permeability leads to dilation of pupils, muscular in coordination, ataxia, muscle tremors and affected insect become paralyzed and stop feeding shortly after exposure. Fang et al., (2002) who reported that spinosad was effective against Sitophilus oryzae and Tribolium castaneum on wheat seeds. Also Rathod et al., (2018) opined that spinosad45 SC @ 4.4 mg per kg of seed were found equally effective for control of pulse beetle in pigeon pea seed after 6 months of storage.

Similarly, Ajaykumara et al., (2017) recorded that emamectin benzoate $5 \mathrm{SG}$ treated seed was found free from the seed damage of $R$. dominica after 6 months of storage.

\section{Seed weight loss}

Up to ninety days after treatment all the seed protectant chemicals afforded complete protection against damage by $S$. cerealella resulting in without any seed weight loss.

After one hundred and eighty days after treatment, spinosad $45 \mathrm{SC}$ recorded lowest per cent weight loss $(0.23 \%)$ followed by emamectin benzoate 5SG $(0.47 \%)$ treated seeds, deltamethrin $2.8 \mathrm{EC}(1.40 \%)$, acorus TNAU formulation (1.53\%), sweet flag rhizome powder (1.86\%) and chlorfenapyr 10 EC $(2.02 \%)$ and highest $(16.68 \%)$ per cent weight loss was recorded in untreated control which was found to be significantly inferior to all the treatments.

The weight loss of the grain was positively and significantly correlated with the per cent damage and number of moths emerged. Better seed quality was observed with seed protectant chemicals because of lower insect infestation noticed with these treatments.

The present findings are in conformity with Antoine et al., (2010) who found the effectiveness of spinosad as grain protectant in controlling C. maculatus even after six months of storage and Kalasagonda (1998) reported that there was no weight loss in wheat grains treated with sweet flag rhizome powder at 0.8 per cent concentration (Fig. 1 and Table 2). 


\section{Adults emerged/ $100 \mathrm{~g}$ of seeds}

With respect to presence of live adults, no population was recorded in any treatment at ninety days after treatment imposition. Observations recorded at one hundred and eighty days after treatment imposition revealed that among the different seed protectant chemicals minimum population build up was noticed in spinosad $45 \mathrm{SC}$ (1 adult $/ 100 \mathrm{~g}$ seeds) followed by emamectin benzoate 5 SG (2 adults /100g seeds) both these are considered as best treatments and superior over rest of the treatments (Table 3). Whereas, the highest $(32.33$ adults $/ 100 \mathrm{~g}$ of seeds) adult emergence was noticed in untreated control (Fig. 2).

Exposure to spinosad produces involuntary muscle contractions followed by paralysis. This suggests that the chemical interacts with the nervous system, causing over excitement and death of insects.

Monsoon (2016) reported lowest seed emergence of $S$. cerealella in spinosad treated bags (2.67 adults / 100g seeds) after six months after storage. Similarly Sharma and Michaelraj (2006) evaluated spinosad as seed protectant against $R$.dominica @ 0.5, 1.0 and $2.0 \mathrm{mg}$ a.i. per $\mathrm{kg}$ of seed and observed complete mortality at all the doses tested for four months without any damage to the seeds. Daglish and Nayak (2006) opined that spinosad applied at 0.5 to $1 \mathrm{mg}$ per $\mathrm{kg}$ was completely effective for nine months, with 100 per cent adult mortality of $R$. domnica after 14 days of exposure to treated seeds and no live F1 adults produced.

In evaluation of bioefficacy seed protectant chemicals, spinosad 45 SC and emamectin benzoate $5 \mathrm{SG}$ were effective in reducing seed damage, seed weight loss and population buid up and afforded the complete protection against angoumois grain moth up to one hundred and eighty days after treatment. Hence, the use of seed protectants is a common preventive measure to protect store grain from insect damage and maintain the quality of seeds.

\section{References}

Ajaykumara, K. M., Thirumalaraju, G. T. and Anil, I., 2017, Evaluation of newer molecule insecticides for the management of Rhyzopertha dominica (F.) (Bostrichidae: Coleoptera) and their effect on seed quality in stored maize. Chem. Sci. Rev. Lett., 6(24): 2520-2525.

Anonymous, 2018, Area, production and productivity of rice crop. INDIASTAT.

Antoine, S., Niango, M. B. A., Clementine, L., Dabire, A. and Pittendrigh. A., 2010, Effectiveness of spinosad (Naturalytes) in controlling the cowpea storage pest, Callosobruchus maculatus (Coleoptera: Bruchidae). J. Econ Entomol., 103(1): 203-210.

Athanassiou, C. G., Kavallieratos, N. G., Vayias, B. J., Papagregoriou, A. S., Dimizas, C. B. and Buchelos, C., 2004, Residual toxicity of beta cyflurthrin, alpha cypermethrin and deltamethrin against Tribolium confusum (J.) (Coleoptera: Tenebrionidae) on stored wheat. Appl. Entomol. Zoo., 39: 195202.

Daglish, G. J. and Nayak, M. K., 2006, Longterm persistence and efficacy of spinosad against Rhyzopertha dominica (Coleoptera: Bostrychidae) in wheat, Pest Management Sci., 2006, 62(2): 148-152.

Fang, L., Subramanyam, B. and Dolder S., 2002, Persistence and efficacy of spinosad residues in farm stored wheat. J. Econ. Entomol., 95(5): 1102-1109.

Harris, K. L. and Lindblad, C. J., 1978, A 
manual of methods for the evaluation of post harvest losses. Am. Assoc. Cereal. Chem., 21(9): 75-79.

Kalasagond, P. R., 1998, Management of beetle pests in stored wheat by non insecticidal approaches. M. Sc. (Agri.) Thesis, Uni. of Agric. Sci., Dharwad (India), pp. 132.

Mohan, S. and Kavitharaghavan, Z., 2008, Studies on the popularization of TNAU stored product insect management kit technology. Green Farming, 6: 53.

Monsoon, K., 2016, Effect of carbon dioxide and insecticides against Angoumois grain moth, Sitotroga cerealella (OLIVIER) in Maize. M. Sc. Thesis, PJTSAU, Hyderabad.

Rathod, P. K., Kolhe, G. K., Chopade, B. J., Borkar, P. A., Murumkar, R. P., Bhalkare, S. K. and Mate, V. N., 2018, Effect of newer insecticide Molecules on Seed Viability, Int. J. Curr. Microbiol. Appl. Sci., 6: 2561-2566.

Sharma, R. K. and Michaelraj, S., 2006, Efficacy of spinosad as seed protectant against the pests of stored maize. $J$. Pesticide Res., 18(2): 173-176.

\section{How to cite this article:}

Basavanjali, Sushila Nadagouda, A. Prabhuraj, Shivaleela and Basavegowda. 2019. Bioefficacy of Seed Protectant Chemicals for Management of Angoumois Grain Moth on Paddy. Int.J.Curr.Microbiol.App.Sci. 8(10): 626-633. doi: https://doi.org/10.20546/ijcmas.2019.810.071 